\title{
'Sense of belonging': The influence of individual factors in the learning environment of South African interns
}

\author{
K L Naidoo, ${ }^{1,2}$ MB ChB, DCH, FCPaed; J van Wyk, ${ }^{3}$ BSc Ed, BEd, MEd, PhD; M Adhikari, ${ }^{2}$ MB ChB, FCPaed, PhD \\ ${ }^{1}$ King Edward VIII Hospital, KwaZulu-Natal Department of Health, Durban, South Africa \\ ${ }^{2}$ Department of Paediatrics and Child Health, Nelson R Mandela School of Medicine, University of KwaZulu-Natal, Durban, South Africa \\ ${ }^{3}$ Department of Clinical and Professional Practice, Nelson R Mandela School of Medicine, University of KwaZulu-Natal, Durban, South Africa
}

Corresponding author: KNaidoo (naidook9@ukzn.ac.za)

Background. The focus is usually on organisational issues when reporting factors influencing the perceptions of South African (SA) medical interns regarding their learning environment (LE). Individual demographic factors are now being recognised as equally important in influencing these perceptions.

Objective. To determine whether individual demographic factors influence interns' perceptions of the LE during their paediatrics rotation in hospitals burdened with high disease in SA.

Methods. Perceptions of the LE among interns in KwaZulu-Natal, SA, were assessed in December 2015, using a validated version of the Postgraduate Hospital Educational Environmental Measure (PHEEM). Overall and subscale PHEEM scores were calculated using Likert scales. The association of these scores with various sociocultural factors relevant to the SA context, previous educational exposure and year of internship were examined using ANOVA or Student $t$-tests.

Results. A total of 209 interns (59.3\%) was sampled. The ethnic breakdown of sampled interns reflected the changing demographic profile of SA junior doctors. Statistically significant associations of overall and teaching subscale PHEEM scores were found with ethnicity ( $p=0.024)$, urban/rural status ( $p=0.023$ ), year of internship ( $p=0.0047)$ and university origin $(p=0.015)$. These factors corroborated characteristics that reflect both past disadvantage in the SA context, and those of being an 'outsider' in an established group.

Conclusions. Intern training programmes in SA need to recognise that individual demographic factors influence interns' perceptions in the context of teaching and mentoring in a discipline. With rapid changes in the demographic profiles of junior doctors, SA intern trainers need to enable a 'sense of belonging' in LEs.

Afr J Health Professions Educ 2018;10(1):50-55. DOI:10.7196/AJHPE.2018.v10i1.953

The learning environment (LE) refers to a 'set of factors' that describes the experiences of the trainee within an organisation. ${ }^{[1]}$ These factors can be divided into three components. The first is the 'physical environment' (facilities, comfort, safety and food), which are the organisational aspects. Work load and work hours would also relate to this aspect. ${ }^{[2]}$ The second component is the 'intellectual environment', which includes support provided for scholarly activities during training, learning with patients and using evidence-based knowledge and skills. ${ }^{[2]}$ The third component is the 'emotional environment', referring to the social support provided, the levels of harassment experienced by the trainee and the trainee's characteristics that may facilitate or hinder access to support, including that offered by a supervisor. ${ }^{[2]}$ The effect of the LE appears to be mediated by the trainees' own perceptions thereof, and this has been shown to be an important determinant of attitude, satisfaction and achievements. ${ }^{[3,4]}$ An optimally functioning clinical LE, where medical interns perceive it as such, is important for successful training in any platform to develop competent physicians. ${ }^{[5]}$

Emphasis has previously been placed on evaluating the organisational aspects of these training platforms. ${ }^{[6,7]}$ The environment in which South African (SA) medical interns train has been associated with excessive workloads, long hours, high stress levels, burnout and reports of suboptimal supervision. ${ }^{[8-10]}$ While these organisational aspects form a significant segment of the factors affecting perceptions of the LE, the influence of individual demographic factors also needs to be explored and understood. ${ }^{[11]}$

The legacy of apartheid policies and persistent social inequity in SA has continued to manifest in society, including in education. ${ }^{[12]}$ The characteristics linked to social inequity, such as gender, ethnicity and socioeconomic status, have persisted, and remain useful as criteria to measure previous disadvantage. Urban/rural status still reflects racial and socioeconomic divisions, and plays an important role in access to and success in higher education. ${ }^{[12]}$ Previous educational experiences are considered important contextual factors in learning and in the SA context; huge disparities exist between the education offered by fee-paying compared with non-fee-paying schools. ${ }^{[13]}$

In the higher-education climate, including health professions education in SA, calls are being made to challenge and dismantle the colonial curricula mindsets that perpetuate the ideological framework that allows one culture to dominate others. ${ }^{[14]}$ While these calls for 'decolonisation' include aspirations for the 'creation of a humanising culture of practice that is not at odds with lived practice, education processes are still noted to have a 'mandated ignorance', with LEs seemingly blind to issues of race and difference. ${ }^{[15]}$ 
Demographic factors are being recognised as important indicators of inequity that can be used for its redress, and most SA medical schools have amended their undergraduate selection criteria towards transformation norms. ${ }^{[13]}$ This has seen a rapid change in the demographic composition of the intern population in SA. As a result of these changes, interns of differing socioeconomic and educational backgrounds are allocated to work and learn together in regional hospitals throughout the country for a 2-year internship.

The internship programme includes all major medical specialties, including paediatrics. ${ }^{[16]}$ It is not clear how the changes in the composition of the group of newly qualified doctors have influenced their perceptions of the LE, especially in paediatrics. An improved understanding of these changes would facilitate the improvement of training for junior doctors. The Postgraduate Hospital Educational Environmental Measure (PHEEM) is a well-recognised instrument used internationally to assess the LE in postgraduate medicine. ${ }^{[17-19]}$ A local SA version of the PHEEM instrument was validated among a cohort of paediatric interns in four hospital complexes in Durban and Pietermaritzburg, KwaZulu-Natal (KZN). ${ }^{[18]}$

While organisational and institutional factors were identified as obstacles to creating an ideal LE, significant differences were noted in the way interns and their supervisors perceived the LE, especially with regard to supervision and mentoring. ${ }^{[18]}$ In this study, we report on the influence of individual demographic factors on perceptions of the LE among this cohort. This study was thus conducted to:

(i) determine whether individual demographic factors influence interns' perceptions of their experiences in the LE in paediatrics;

(ii) compare the perceptions of first- and second-year interns of the LE in the paediatric rotation; and

(iii) determine the influence of previous educational experiences on paediatric interns' perceptions of their LE.

\section{Methods}

\section{Research design and ethics approval}

This was an observational, cross-sectional cohort study. Ethical approval for the study was obtained from the University of KZN Biomedical Research Ethics Committee (ref. no. BE 177/15), and gatekeepers' permission was granted from the various institutions, as well as the Health Research and Knowledge Management subcomponent of the Department of Health in the province of KZN. The study population consisted of all eligible interns, who were informed of the study and invited to participate. Participants were informed of their rights, and could withdraw at any stage. Participation in the study was voluntary, and the anonymity and confidentiality of respondents were assured. The surveys were group-administered at preexisting intern meetings, and the primary researcher was blinded to the individual responses as no identifying details were required.

\section{The instrument}

The PHEEM has been used to assess the LE among interns throughout the world. ${ }^{[17]}$ The PHEEM used in our study had eight minor changes made to the original 40 items to accommodate terminology relevant to the SA and paediatric setting. ${ }^{[18]}$ Each item was scored by participants on a fivepoint Likert scale, where 1 indicated 'strongly disagree' and 5 represented a 'strongly agree' response. The original questionnaire used a 0 - 4 scale, while we followed a more conventional scale of $1-5$, as used by some authors in clinical settings. ${ }^{[3]}$

\section{Procedure}

The sample population included all interns who had completed a paediatrics rotation at four hospital complexes (comprising eight hospitals) in both major cities of the KZN province in December 2015. Demographic data, including gender, ethnicity, home language, urban/rural status and the highest educational level of a 'parental figure' were obtained. Three categories of urban/rural status were recognised, namely urban (mainly city and suburban neighbourhoods), semi-urban (reflecting mainly 'township' neighbourhoods) and rural (mainly outside of an urban or semi-urban area). These distinctly different area types reflect significant racial and socioeconomic divisions in the SA context. ${ }^{[12]}$ Data on prior educational exposure were also solicited, on the type of high school attended (whether fee-paying or non-fee-paying) and university origin (whether the intern graduated from the local university (University of KwaZulu-Natal) or from another university in a different province or country), and on interns' finalyear undergraduate paediatric performance.

\section{Sample size}

A sample-size calculation was based on the comparison of the PHEEM scores between the intern group and various demographic variables. Using a one-way ANOVA with up to four groups, the sample size of 209 interns was found to be adequate, as a sample of 180 was required to achieve $80 \%$ power at a $5 \%$ significance level. ${ }^{[20]}$

\section{Data analysis}

The overall PHEEM scale and subscale scores were calculated for each participant. Where there were missing data, means were computed based on data for available items, provided this did not exceed $20 \%$ of the items. The overall score was computed as the average of all 40 items. The negatively worded items 7, 8, 11 and 13 were reverse-scored.

For the descriptive analysis, categorical variables were summarised by frequency and percentage tabulation. Continuous variables were summarised by mean, standard deviation, median and interquartile range. The association between the various demographic variables, year of internship and the factors associated with previous educational experiences, with overall PHEEM score as well as the three subscale PHEEM scores, was determined by the $t$-test or ANOVA (for more than two categories). The strength of the association was measured by Cohen's $d$. The following scale of interpretation was used: $\geq 0.8$ = large effect; 0.5 - $0.79=$ moderate effect; and $0.2-0.49=$ small effect. Data analysis was carried out using SAS Version 9.4 for Windows (SAS, USA). The $5 \%$ significance level was used throughout.

\section{Results}

A response rate of $59.3 \%$ was achieved, as 209 completed questionnaires were returned from a potential pool of 352 interns. Of these, $35.8 \%$ of the interns assessed were in their first year and $63.8 \%$ in their second year of internship, and $55 \%$ were female. The mean age of the whole group was 26.2 years (standard deviation (SD) 2.6; range 20 - 37 years). A number of factors were examined to investigate the influence of previous disadvantage on perceptions. Table 1 presents the sociocultural characteristics of the sampled interns. Table 2 shows the composition of sampled interns with regard to variables indicating previous educational experiences.

An examination of the influence of various demographic factors on the overall PHEEM scores indicated a number of significant findings. Table 3 depicts the 


\begin{tabular}{|c|c|}
\hline Variable & $n(\%)$ \\
\hline \multicolumn{2}{|l|}{ Gender } \\
\hline Male & $91(45.05)$ \\
\hline Female & $111(54.95)$ \\
\hline \multicolumn{2}{|l|}{ Ethnicity } \\
\hline White & $53(27.89)$ \\
\hline Indian & $64(33.68)$ \\
\hline Black African & $60(31.58)$ \\
\hline Coloured & $13(6.84)$ \\
\hline \multicolumn{2}{|l|}{ Home province } \\
\hline KZN & $112(66.67)$ \\
\hline Gauteng & $27(16.07)$ \\
\hline Western Cape & $23(13.69)$ \\
\hline Eastern Cape & $6(3.57)$ \\
\hline \multicolumn{2}{|l|}{ Urban/rural status } \\
\hline Urban & $119(58.91)$ \\
\hline Semi-urban (township) & $63(31.19)$ \\
\hline Rural & $20(9.90)$ \\
\hline \multicolumn{2}{|l|}{ Home language* } \\
\hline English & $105(62.87)$ \\
\hline Afrikaans & $29(17.37)$ \\
\hline Zulu & $25(14.97)$ \\
\hline Xhosa & $8(4.79)$ \\
\hline \multicolumn{2}{|c|}{ Highest achieved educational level of parent/caregiver } \\
\hline Less than high school completion & $15(7.54)$ \\
\hline Completed high school & $17(8.54)$ \\
\hline Non-university tertiary & $33(16.58)$ \\
\hline University & $134(67.34)$ \\
\hline
\end{tabular}

\begin{tabular}{ll}
\multicolumn{2}{l}{ Table 2. Previous educational experience of sampled interns $(\mathbf{N = 2 0 9 )}$} \\
\hline Variable & $n(\%)$ \\
\hline High school type & \\
$\quad$ Non-fee-paying government & $67(43.50)$ \\
$\quad$ Fee-paying government & $63(31.50)$ \\
$\quad$ Fee-paying private & $50(25.00)$ \\
University origin: local (UKZN) v. non-local (all other) & \\
$\quad$ Local & $60(29.56)$ \\
$\quad$ Non-local & $143(70.44)$ \\
University origin: SA v. non-SA & \\
$\quad$ SA & $162(81.00)$ \\
$\quad$ Non-SA & $38(19.00)$ \\
Undergraduate paediatrics performance & \\
$\quad<60 \%$ pass & $19(9.45)$ \\
$60 \%$ - 70\% pass & $112(55.72)$ \\
$>70 \%$ pass & $70(34.83)$ \\
UKZN = University of KwaZulu-Natal. &
\end{tabular}

relationship between all the individual demographic variables, including sociocultural factors, factors indicating previous educational experience and internship year, and the overall PHEEM score.
Table 3. Comparisons of the overall mean PHEEM scores with all demographic variables

\begin{tabular}{|c|c|c|}
\hline Sociocultural factors & $n$, mean (SD) & $p$-value ${ }^{*}$ \\
\hline \multicolumn{3}{|l|}{ Gender } \\
\hline Male & $90,3.48(0.48)$ & \multirow{2}{*}{0.59} \\
\hline Female & $109,3.52(0.52)$ & \\
\hline \multicolumn{3}{|l|}{ Ethnicity } \\
\hline White & $53,3.57(0.36)$ & \multirow{4}{*}{0.024} \\
\hline Indian & $64,3.55(0.55)$ & \\
\hline Black African & $57,3.37(0.55)$ & \\
\hline Coloured & $13,3.78(0.27)$ & \\
\hline \multicolumn{3}{|l|}{ Home province } \\
\hline KZN & $11,3.52(0.54)$ & \multirow{4}{*}{0.68} \\
\hline Gauteng & $26,3.64(0.26)$ & \\
\hline Western Cape & $23,3.50(0.38)$ & \\
\hline Eastern Cape & $6,3.46(0.34)$ & \\
\hline \multicolumn{3}{|l|}{ Urban/rural status } \\
\hline Urban & $117,3.59(0.45)$ & \multirow{3}{*}{0.023} \\
\hline Semi-urban (township) & $62,3.37(0.60)$ & \\
\hline Rural & $20,3.50(0.44)$ & \\
\hline \multicolumn{3}{|l|}{ Home language $^{\dagger}$} \\
\hline English & $105,3.55(0.48)$ & \multirow{4}{*}{0.16} \\
\hline Afrikaans & $29,3.64(0.35)$ & \\
\hline Zulu & $24,3.40(0.59)$ & \\
\hline Xhosa & $8,3.29(0.48)$ & \\
\hline \multicolumn{3}{|c|}{ Highest level of education of parent/caregiver } \\
\hline Less than high school completion & $15,3.43(0.81)$ & \multirow{4}{*}{0.66} \\
\hline Completed high school & $17,3.40(0.50)$ & \\
\hline Non-university tertiary & $32,3.51(0.50)$ & \\
\hline University & $1333.54(0.48)$ & \\
\hline \multicolumn{3}{|l|}{ Internship year } \\
\hline First year & $72,3.37(0.56)$ & \multirow{2}{*}{0.0047} \\
\hline Second year & $126,3.58(0.47)$ & \\
\hline \multicolumn{3}{|l|}{ High school attended } \\
\hline Non-fee-paying government & $85,3.49(0.55)$ & \multirow{3}{*}{0.61} \\
\hline Fee-paying government & $62,3.49(0.51)$ & \\
\hline Fee-paying private & $50,3.57(0.48)$ & \\
\hline \multicolumn{3}{|c|}{ University origin: local (UKZN) v. non-local } \\
\hline Local (UKZN) & $59,3.64(0.56)$ & \\
\hline Non-local & $141,3.45(0.49)$ & \\
\hline \multicolumn{3}{|l|}{ University origin: SA v. non-SA } \\
\hline SA & $160,3.54(0.50)$ & \multirow{2}{*}{0.094} \\
\hline Non-SA & $37,3.38(0.53)$ & \\
\hline \multicolumn{3}{|l|}{ Undergraduate paediatric pass mark } \\
\hline$>70 \%$ & $69,3.51(0.45)$ & \\
\hline $60 \%-70 \%$ & $110,3.50(0.55)$ & 0.99 \\
\hline$<60 \%$ & $19,3.50(0.54)$ & \\
\hline
\end{tabular}

\section{Sociocultural variables}

There was a significant association between ethnicity and the overall PHEEM score. Interns who had self-identified as black African had lower 


\section{Research}

mean PHEEM scores than their white, Indian or coloured colleagues. This finding was reiterated when comparing ethnicity with PHEEM scores on the teaching subscale ( $p=0.0026$ ) (Table 4 ). The effect size was large when comparing the scores of coloured $(d=0.88)$ and Indian $(d=0.5)$ with black African interns.
There was a significant association between the mean PHEEM score and urban/rural status, with those who indicated that they came from a semiurban (mainly referring to a 'township' area) environment having a lower overall PHEEM score than those from urban (city or suburbs) rural areas

Table 4. Comparison of PHEEM teaching subscale scores with all demographic variables

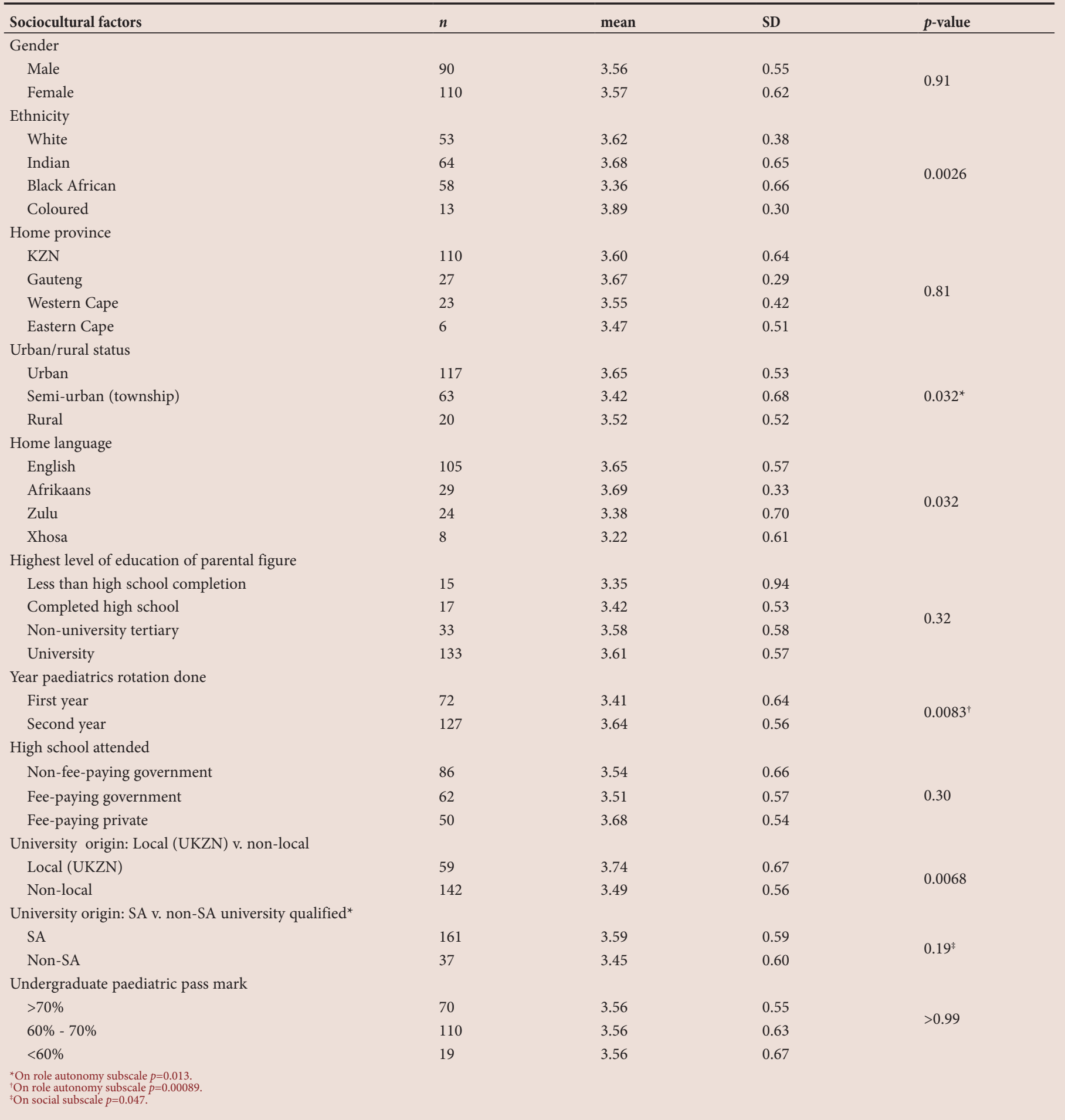


(Table 3). There were statistically significant associations between urban/ rural status and the mean PHEEM score on the teaching subscale $(p=0.032)$ and the PHEEM score on the role-autonomy subscale ( $p=0.013$ ) (Table 4 ).

Table 4 shows the mean PHEEM teaching subscale scores compared with the major languages spoken by interns, which also showed a significant association $(p=0.032)$.

We found no statistically significant relationship with gender, home province or the highest educational level of an intern's parental figure, when comparing overall PHEEM scores and all subscale scores with these sociocultural variables. There were no significant associations when we compared the PHEEM subscale scores on the social-support scales with all sociocultural variables.

\section{Internship year}

The mean PHEEM score for interns in their first year was significantly lower than that of interns in their second year of internship. This significant difference between year 1 and 2 interns was seen when comparing PHEEM scores on the teaching subscale $(p=0.0083)$ (Table 4$)$, as well as on the PHEEM role-autonomy subscale scores $(p=0.0089)$.

\section{Prior educational exposure}

Table 4 indicates that interns who had graduated from the local university had significantly higher perceptions of the LE than interns who had graduated outside the province. There was a significant association between the mean PHEEM scores of interns who studied overseas, and SA-trained interns, on the social support subscale score. Neither the type of high school attended nor undergraduate performance in paediatrics showed any statistically significant relationship with overall PHEEM score or with the PHEEM scores on the teaching, role-autonomy and social support subscales.

\section{Discussion}

In this study, individual demographic factors are shown to have a major impact in influencing interns' perceptions of the LE. These characteristics have been largely neglected as factors to consider in influencing internship, while organisational factors such as work-hours and the state of the physical infrastructure of the LE have been focused on.

The good response rate in our sample was in keeping with surveys using the PHEEM instrument elsewhere, ${ }^{[17]}$ and the distribution of sampled interns closely represented the allocation of interns across the hospital complexes. The 2015 cohort shows an increasing representation of female and black African newly qualified doctors compared with previous years, and is beginning to reflect the implementation of amended selection criteria at SA medical schools. ${ }^{[13]}$ However, evaluating the demographic characteristics of the sampled interns revealed that the newly qualified doctors are still largely drawn from middle-class backgrounds, with nearly $60 \%$ of interns originating from urban areas, $56.5 \%$ attending fee-paying schools and over $60 \%$ from homes with at least one parental figure having obtained a university qualification.

In this study, ethnicity, language and urban/rural status were identified as factors that are significantly associated with lower perceptions of the LE in internship. These relationships corroborate the notion that interns from previously disadvantaged communities have poorer perceptions of the LE than most of their peers in internship. Gender was not identified as a factor influencing perceptions of the LE. Paediatrics is generally a discipline with a larger female composition, and thus probably reflects a more gender- sensitive environnment. Various other studies have shown the influence of gender on the overall PHEEM scores, especially in disciplines with an underrepresentation of female doctors such as general surgery and intensive care. ${ }^{[21,22]}$

This study showed a clear difference in the perceptions of interns who were in their first year as compared with those in the second year of internship. Various studies internationally corroborate this finding, with juniors having less positive perceptions of the LE than senior trainees. ${ }^{[23]}$ Interns who did not graduate from the university supporting the internship training platform, and those who graduated outside SA, also displayed poorer perceptions of the LE. These findings are consistent with the findings of studies that reported higher levels of stress among interns at hospitals in SA who graduated from non-local universities. ${ }^{[9]}$

The findings show that while indices of socioeconomic disadvantage, especially ethnicity and urban/rural status, did influence interns' perceptions of the LE, these were not the only factors. The combination of factors that significantly influenced the perceptions of the LE relate to characteristics of being 'new' or 'different' to the established norms or 'culture'. The factors can furthermore be categorised as individual characteristics that seemingly add to perceptions of marginalisation or 'alienation' in interns who experienced their training as 'being isolated from a group activity in which they should be involved.'[24] This difficulty in developing a 'sense of belonging' is of concern, especially as learning within the clinical environment relies heavily on participation within a 'community of practice' that is provided by the authentic work environment.

Learning in internship occurs within the context of 'legitimate, peripheral participation' within a social context, and is an increasingly communal and negotiated contract. ${ }^{[25,26]}$ This learning, while understood at an individual level, occurs at group level, and involves the acquisition of knowledge, skills, attributes, values and competencies and 'participation in social processes' where learning is inextricably linked to and embedded in its context. ${ }^{[27]}$

The interaction between supervisors and interns occurs within a historic context, and reflects a 'colonised' culture where the relationship of dominance creates the concept of the 'outsider, ${ }^{[28]}$ and in new incumbents, a notion of being the 'other' or not being welcome in an already established setting that does not recognise his/her presence. These unequal power relations and marginalisation may contribute to a failure to ensure that all interns are brought into full participation. ${ }^{[14]}$ The learning climate, in this context, may thus be difficult for interns who experience it from the position of 'outsiders' or 'others', and they are unlikely to seek or initiate a search for effective mentorship, a situation that further compromises the supervision opportunity.

These findings, which are of relevance to all health professionals and to undergraduate universities, indicate the need for efforts to ensure that all interns develop a 'sense of belonging' in their training platform. The impact of the findings on policy includes the recognition by intern accreditation bodies of the role of individual intern characteristics as important factors to consider when developing intern training curricula and oversight frameworks. This can translate into processes to mandate the development of welcoming environments that facilitate the integration of interns from the start, so that they commence as a team respecting and appreciating each other's contexts and diversity. The calls to work as a collective to 'decolonise' and humanise training have resonance here. ${ }^{[14]}$ Intern programmes and curricula need to change to evaluate intern and supervisor interactions, to ensure that all interns experience optimal supervision and that individual demographic factors are taken into consideration. 
As the SA medical community transforms to reflect the true demographics of the country, efforts should be made to ensure the inclusion of and support for 'engagement' of junior doctors within hierarchical and 'established' communities of practice. Individual demographic factors in the LE can no longer be regarded as minor factors in the learning process, and more work is needed to understand how they impact on successful orientation and learning, especially in the formative period of internship.

\section{Limitations}

The sample only included interns linked to hospitals in one province, and the possibility exists that the findings could reflect a regional bias. However, the large number of interns sampled, the good response rate and the choice of large hospitals with known high disease burdens is thought to be adequately representative of the SA internship programme, and adds confidence that the findings would probably represent those of others, including other health professionals, in the SA setting.

The PHEEM instrument was originally created for postgraduate registrars; however, we believe that the LE of interns' work resembles that of the postgraduate registrar trainees, and PHEEM is therefore highly relevant.

This study did not explore the training received or the previous clinical experience of supervisors, which would influence the mentorship relationship in this setting.

This study used quantitative methods to assess the LE and to fully understand the LE in depth; a qualitative evaluation of interns' perceptions of the LE is also needed.

\section{Conclusion}

While organisational factors have been noted to affect the LE of interns in SA, our research indicates that individual demographic factors are important. Perceptions of the LE, as measured by validated and reliable tools like the PHEEM, are influenced by various demographic and individual factors. First-year interns who have not graduated from the local university and who are from previously disadvantaged socioeconomic groups in SA are more likely to perceive a poorer LE than their peers. These factors affecting a 'sense of belonging' will become apparent in challenged situations where there is inadequate supervision and mentoring, and within the rapidly transforming demographic environment in SA as it attempts to 'decolonise' its practices.

Efforts must be made to ensure that medical-intern and all healthprofessional training policies and practices recognise that these factors must be considered during teaching, mentoring and supervision. Further qualitative studies into these relationships are needed to improve our understanding in clinical settings as we aim to train competent health professionals for effective practice in transformed settings.

Acknowledgements. The authors would like to express their gratitude to the interns and intern supervisors in the KZN hospitals who participated in this study, Dr Petra Gaylard (DMSA) for assistance with the statistical analyses and Mrs Leora Sewnarain for assistance with typesetting and formatting.
Author contributions. KLN was responsible for study design, data collection, data analysis and drafting the manuscript. JVW was responsible for supervision of the entire work, study design and manuscript review. MA was responsible for supervision of the entire work and manuscript review.

Funding. MEPI Funding: This publication was made possible by grant number R24TW008863 from the Office of the US Global AIDS Co-ordinator and the US Department of Health and Human Services, National Institutes of Health (Office of AIDS Research and Office of Research on Women's Health). Its contents are solely the responsibility of the authors and do not necessarily represent the official views of the US government.

Conflicts of interest. None.

1. Wall D, Clapham M, Riquelme A, et al. Is PHEEM a multi-dimensional instrument? An international perspective. Med Teach 2009;31:e521-e527. https://doi.org10.1186/1472-6920-14-226

2. Mohanna K, Cottrell E, Wall D, Chambers R. Teaching Made Easy: A Manual for Health Professionals. 1st ed. Boca Raton: CRC Press, 2010

3. Boor K, Scheele F, van der Vleuten C, et al. Psychometric properties of an instrument to measure the clinical learning environment. Med Educ 2007;41(1):92-99. https://doi.org/10.1111/j.1365-2929.2006.02651.x

4. Genn, J. AMEE Medical education guide no. 23 (Part 1): Curriculum, environment, climate, quality and change in medical ducation - a unifying perspective. Med Teach 2001;23(4):337-344. https://do org $/ 10.1080 / 01421590120063330$

5. Hoff T, Pohl $\mathrm{H}$, Bartfield J. Creating a learning environment to produce competent residents: The roles of culture and context. Acad Med 2004;79(6):532-540. https://doi.org/10.1097/00001888-200406000-00007

6. Sein N, Tumbo J. Determinants of effective medical intern training at a training hospital in North West Province, South Africa. Afr J Health Professions Educ 2012;4(1):10-14.

7. Hospital reform and staff morale in South Africa: A case study of Dr Yusuf Dadoo Hospital. S Afr Fam Pract 2013;55(2):180-185. https://doi.org/10.1080/20786204.2013.10874330

8. Erasmus N. Slaves of the state - medical internship and community service in South Africa. S Afr Med J Erasmus N. Slaves of the state - medical internship
2012;102(8):655-658. https://doi.org/10.7196/samj.5987

9. Sun GR, Saloojee H, Jansen van Rensburg M, Manning D. Stress during internship at three Johannesburg hospitals. S Afr Med J 2008;98(1):33-35.

10. Bateman C. System burning out our doctors - study. S Afr Med J 2012;102(7):593-594.

11. Tyssen R, Vaglum P, Grønvold N, Ekeberg $\emptyset$. The relative importance of individual and organisational factors for the prevention of job stress during internship: A nationwide and prospective study. Med Teach 2005;27(8):726-731 https://doi.org/10.1080/01421590500314561

12. Statistics South Africa. Census 2011 Statistical release. Pretoria: StatsSA, 2012. http://www.statssa.gov.za publications/P03014/P030142011.pdf (accessed 23 January 2017).

13. Van der Merwe L, Van Zyl G, St Clair Gibson A, et al. South African medical schools: Current state of selection criteri and medical students' demographic profile. S Afr Med J 2016;106(1):76-81. https://doi.org/10.7196\%2FSAMJ.2016. v106i1.9913

14. Pillay M, Kathard H. Decolonising health professionals' education: Audiology and speech therapy in South Africa. Afr J Rhetoric 2015;7(1):193-227.

15. Brydon D, Dvořák M. Crosstalk: Canadian and Global Imaginaries in Dialogue. Waterloo: Wilfrid Laurie University Press, 2012.

16. Medical and Dental Professions Board, Health Professions Council of South Africa. Handbook on Internship Training: Guidelines for Interns, Accredited Facilities and Health Authorities. Pretoria: HPCSA, 2016.

17. Soemantri D, Herrera C, Riquelme A. Measuring the educational environment in health professions studies: A Soemantri D, Herrera C, Riquelme A. Measuring the educational environment in health profe
systematic review. Med Teach 2010; 32(12):947-952. https://doi.org/10.3109/01421591003686229

18. Naidoo KL, van Wyk JM, Adhikari M. The learning environment of paediatric interns in South Africa. BMC Med Educ 2017;17(1): 235. https://doi.org/10.1186/s12909-017-1080-3

19. Roff S, McAleer S, Skinner A. Development and validation of an instrument to measure the postgraduate clinical learning and teaching educational environment for hospital-based junior doctors in the UK. Med Teach 2005;27(4):326-331. https://doi.org/10.1080/01421590500150874

20. Faul F, Erdfelder E, Lang A, Buchner A. G*Power 3: A flexible statistical power analysis programme for the social, behavioral, and biomedical sciences. Behav Res Methods 2007;39(2):175-191. https://doi.org/10.3758/bf03193146 1. Kanashiro J, McAleer S, Roff S. Assessing the educational environment in the operating room - a measure of resident perception at one Canadian institution. Surgery 2006;139(2):150-158. https://doi.org/10.1016/j.surg.2005.07.005

22. Clapham M, Wall D, Batchelor A. Educational environment in intensive care medicine - use of Postgraduate Hospital Educational Environment Measure (PHEEM). Med Teach 2007;29(6):e184-e191. https://doi. Hospital Educational Environ
org/10.1080/01421590701288580

23. Pinnock R, Reed P, Wright M. The learning environment of paediatric trainees in New Zealand. J Paediatr Child Health 2009;45(9):529-534. https://doi.org/10.1111/i.1440-1754.2009.01553.x

24. Mann S. Alternative perspectives on the student experience: Alienation and engagement. Stud High Educ 2001;26(1):7-19. https://doi.org/10.1080/03075070020030689

25. Lave J, Wenger E. Legitimate peripheral participation in communities of practice. In: Cross RL, Israelit SB, eds. Strategic Learning in a Knowledge Economy. Boston: Butterworth-Heinemann, 2000:167-182.

26. Mann K. Theoretical perspectives in medical education: Past experience and future possibilities. Med Educ 2010;45(1):60-68. https://doi.org/10.1111/j.1365-2923.2010.03757.x

27. Yardley S, Teunissen P, Dornan T. Experiential learning: AMEE Guide No. 63. Med Teach 2012;34(2):e102-e115. https://doi.org/10.3109/0142159X.2012.650741

28. Spivak GC. A Critique of Postcolonial Reason. Harvard: Harvard University Press, 1999. 